\section{OP0246 MIR-214-3P PROTECTS AGAINST OSTEOARTHRITIS BY DIRECTLY TARGETING NF-KB PATHWAY}

Y. Cao ${ }^{1}$, S. Tang ${ }^{1}$, X. $\mathrm{Nie}^{1}$, W. $\mathrm{Han}^{1}$, Z. Zhu ${ }^{1}$, C. Ding ${ }^{1} .{ }^{1}$ Clinical Research Centre, Zhujiang Hospital, Southern Medical University, Guangzhou, China, GuangZhou, China

Background: Osteoarthritis $(O A)$ is a degenerative disease associated with changes in the articular cartilage and bone, severely affecting patients' mobility and quality of life. Multiple factors including mechanical stress, metabolic alteration and inflammatory mediators are involved in the complex pathogenesis of $O A[1]$. Interventions targeting these pathogenic factors may contribute to the treatment of OA. MiRNAs are single strand non-coding small RNAs, which are regulated in chondrogenesis and $\mathrm{OA}[2,3]$. Recent studies demonstrated that miRNAs are involved in the regulation of NF-KB signaling pathway by different mechanisms[4]. These interactions suggest that NF-KB related miRNAs may be used as potential biomarkers and drug therapeutic targets in clinical treatment of OA. However, the relationship between miR-214-3p and NF-kB pathway remains poorly understood in OA.

Objectives: This study aimed to test the expression and biological function of miR-214-3p in OA, and explore its mechanism in osteoarthritic chondrocytes. Methods: Articular primary chondrocytes were isolated from human cartilage samples, which were acquired from patients with end-stage knee OA at the time of total knee replacement surgery $(n=27)$, according to protocols approved by the Ethic Committee of Zhujiang Hospital. Real time PCR (RT-PCR) and in situ hybridization (ISH) were used to detect the expression of miR-214-3p in OA and non-OA cartilage tissues. Interference of miR214-3p was conducted using inhibitor, while overexpression of miR-214-3p was performed with mimics. Metabolism of extracellular matrix was detected by RT-PCR, western blotting and immunofluorescence in vitro. Flow cytometry were conducted to determine cell apoptosis. A luciferase reporter assay, was used to evaluate the interaction between miR-214-3p and its downstream target. Human chondrocytes were cotransfected with miR-214-3p and the IKBKB-overexpressing plasmid to confirm the interaction between miR-214-3p and NF-KB pathway. For in vivo studies, experimental OA was induced in 12-week-old male C57BL/6J mice by destabilization of the medial meniscus (DMM) surgery with miR-214-3p agomir intra-articular (IA) injection (once weekly for 12 days) or by IA injection (once weekly for 12 days) of miR-214-3p antiagomir. Mice were sacrificed 10 weeks after the first IA injection, and subjected to histological analyses.

Results: MiR-214-3p was significantly reduced in human OA cartilage. The decreased expression of miR-214-3p in the OA cartilage tissues was directly associated with excessive apoptosis and imbalance between anabolic and catabolic factors of ECM. Mechanistically, we determined that miR-214-3p directly targeted IKBKB/IKK-b and thereby suppressed the activation of NF-KB pathway. IKBKB overexpression attenuated the inhibitory effect of miR-214-3p on NF-KB pathway. Furthermore, inhibition of miR-214-3p in mice joints triggered spontaneous cartilage loss and OA development, while IA injection of miRNA-214-3p agomir alleviated $\mathrm{OA}$ in the DMM mouse model.

Conclusion: Our results reveal an important role of miR-214-3p in OA progression. MiR-214-3p was down-regulated while IKBKB was upregulated in OA. MiR214-3p inhibits the NF-kB signaling pathway and suppresses the progression of OA through targeting IKBKB. Thus, miR-214-3p maybe a therapeutic target for $\mathrm{OA}$.

References:

[1] Glyn-Jones S, Palmer AJR, Agricola R, Price AJ, Vincent TL, Weinans H, Carr AJ: Osteoarthritis. The Lancet 2015, 386(9991):376-387.
[2] Nugent M: MicroRNAs: exploring new horizons in osteoarthritis. Osteoarthritis and cartilage 2016, 24(4):573-580.

[3] Vicente R, Noel D, Pers YM, Apparailly F, Jorgensen C: Deregulation and therapeutic potential of microRNAs in arthritic diseases. Nature reviews Rheumatology 2016, 12(4):211-220

[4] Xu B, Li YY, Ma J, Pei FX: Roles of microRNA and signaling pathway in osteoarthritis pathogenesis. Journal of Zhejiang University Science $B$ 2016, 17(3):200-208

Disclosure of Interests: None declared

DOI: 10.1136/annrheumdis-2020-eular.5731

\section{Progress in scleroderma and myositis}

\section{OP0247 \\ PERSISTENT PREMATURE MORTALITY GAP IN IDIOPATHIC INFLAMMATORY MYOPATHY: A GENERAL POPULATION-BASED COHORT STUDY}

K. D'silva ${ }^{1}$, L. Lu' ${ }^{2}$, A. Ogdie ${ }^{3}$, A. Aviña ${ }^{2}$, H. Choi' ${ }^{1}{ }^{1}$ Massachusetts General Hospital, Rheumatology, Boston, United States of America; ${ }^{2}$ Arthritis Research Canada, Richmond, Canada; ${ }^{3}$ Hospital of the University of Pennsylvania, Rheumatology, Philadelphia, United States of America

Background: Idiopathic inflammatory myopathy (IIM) is associated with significant premature mortality; however, whether the mortality gap has improved over recent years is unknown.

Objectives: To determine trends in premature mortality in patients with IIM in a large cohort, representative of the United Kingdom (UK) general population. Methods: Using The Health Improvement Network (THIN), an electronic medical record database representative of the UK general population, we identified patients with incident IIM between 18 and 89 years of age (defined by at least one Read diagnosis code for dermatomyositis, polymyositis, or interstitial myositis with at least one year of continuous enrollment in THIN prior to the cohort entry date) and up to 10 controls without IIM matched on age, sex birth year, and database entry year. The cohort was divided in two based on the year of IIM diagnosis: the early cohort (1999-2006) and the late cohor (2007-2014). We calculated adjusted hazard ratios for death using a multivariable Cox-proportional hazards model and adjusted rate differences using an additive hazard model.

Results: The early cohort consisted of 355 patients with IIM and 3182 matched controls, while the late cohort consisted of 396 IIM patients and 3551 matched controls. In both cohorts, IIM patients had excess mortality compared to matched controls [57.4 vs. 15.2 deaths/1000 person-years (PY) in the early cohort and 43.2 vs. 14.1 deaths/1000 PY in the late cohort] (Table). The corresponding multivariate mortality hazard ratios were 2.73 $(95 \% \mathrm{Cl}, 1.85$ to 4.03$)$ vs. $2.61(95 \% \mathrm{Cl}, 1.75$ to 3.89$)$ in the early and late cohorts, respectively ( $p$-value for interaction $=0.63$ ) (Figure). The absolute multivariate mortality differences were $36.6(95 \% \mathrm{Cl}, 20.4$ to 52.8$)$ and 25.8 $(95 \% \mathrm{Cl}, 13.7$ to 37.9$)$ deaths/1000 PY, in the early and late cohorts, respectively ( $p$-value for interaction $=0.24$ ).

Conclusion: In this general population-based cohort study, patients with IIM had over 2.5 times the risk of death compared to matched controls, even after adjusting for comorbidities and medications. Unlike trends seen in rheumatoid arthritis and granulomatosis with polyangiitis, there appears to be no improvement in mortality in IIM in recent years. This highlights the need for improved strategies for the management of patients with IIM and its comorbidities.

Table. Association between idiopathic inflammatory myopathy (IIM) and all-cause mortality according to time period.

\begin{tabular}{|c|c|c|c|c|c|}
\hline & \multicolumn{2}{|c|}{$1999-2006$} & \multicolumn{2}{|c|}{$2007-2014$} & \multirow[b]{2}{*}{$\begin{array}{l}\mathrm{p} \text {-value fo } \\
\text { interaction }\end{array}$} \\
\hline & $\begin{array}{l}\text { IIM cohort } \\
(n=355)\end{array}$ & $\begin{array}{l}\text { Non-IIM cohort } \\
\quad(n=3182)\end{array}$ & $\begin{array}{l}\text { IIM cohort } \\
(\mathrm{n}=396)\end{array}$ & $\begin{array}{l}\text { Non-IIM cohort } \\
\quad(n=3551)\end{array}$ & \\
\hline Follow-up time, years (mean $\pm \mathrm{SD}$ ) & $2.6 \pm 2.1$ & $2.9 \pm 2.1$ & $3.2 \pm 2.4$ & $3.5 \pm 2.4$ & \\
\hline Number of deaths & 53 & 140 & 55 & 177 & \\
\hline Death rate/1000 PY $(95 \% \mathrm{Cl})$ & $57.4(43.0,75.1)$ & $15.2(12.7,17.9)$ & $43.2(32.5,56.2)$ & $14.1(12.1,16.3)$ & \\
\hline Age-, sex-, and entry year-matched hazard ratio $(95 \% \mathrm{Cl})$ & $4.02(2.89,5.59)$ & 1.00 (ref) & $3.43(2.49,4.73)$ & 1.00 (ref) & 0.50 \\
\hline Multivariable-adjusted hazard ratio $(95 \% \mathrm{Cl})^{\star}$ & $2.73(1.85,4.03)$ & 1.00 (ref) & $2.61(1.75,3.89)$ & 1.00 (ref) & 0.63 \\
\hline Age-, sex-, and entry year-matched rate difference/1000 PY (95\% Cl) & $42.2(26.6,57.9)$ & 0.0 (ref) & $29.1(17.5,40.7)$ & 0.0 (ref) & 0.24 \\
\hline Multivariable-adjusted rate difference/1000 PY $(95 \% \mathrm{Cl})$ & $36.6(20.4,52.8)$ & 0.0 (ref) & $25.8(13.7,37.9)$ & 0.0 (ref) & 0.24 \\
\hline
\end{tabular}

* Multivariable models were adjusted for age, sex, entry year, number of GP visits, BMI, smoking status (i.e., non-smokers, ex-smokers, current smokers), alcohol consumption (i.e., non-drinkers, ex-drinkers, current drinkers), comorbidities, and medication use.

PY, person-year; BMI, body mass index; GP, general practitioner 


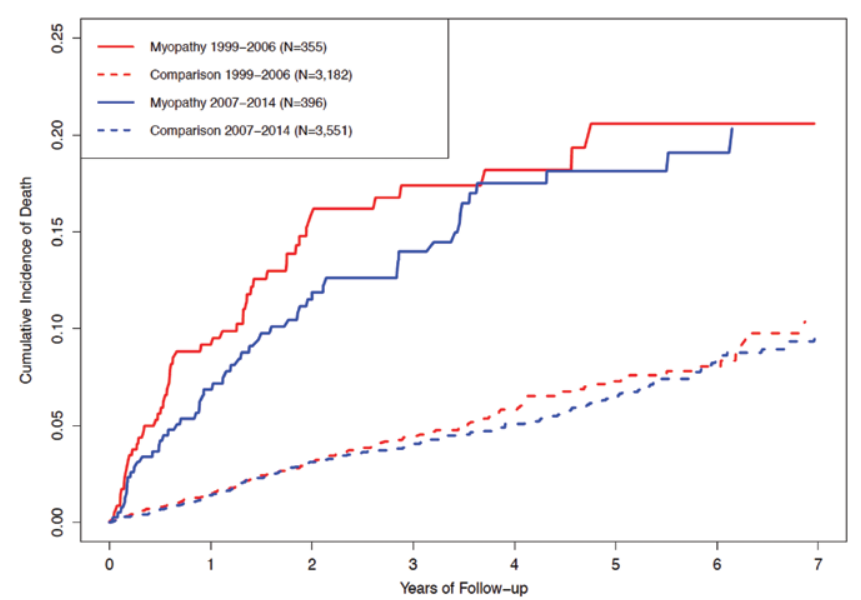

Figure. Cumulative mortality of patients with idiopathic inflammatory myopathy and matched controls without IIM in early versus late cohorts (1999-2006 versus 2007-2014).

Disclosure of Interests: Kristin D'Silva: None declared, Leo Lu: None declared, Alexis Ogdie Grant/research support from: Pfizer to Penn, Novartis to Penn, Amgen to Forward/NDB, Consultant of: Abbvie, Amgen, Bristol-Myers Squibb, Celgene, Corrona, Janssen, Eli Lilly, Novartis, Pfizer, Antonio Aviña: None declared, Hyon Choi Grant/research support from: Ironwood, Horizon, Consultant of: Takeda, Selecta, Horizon, Kowa, Vaxart, Ironwood DOI: 10.1136/annrheumdis-2020-eular.2230

\section{OP0248 $\quad$ PREMATURE MORTALITY BURDEN FOR SYSTEMIC SCLEROSIS: NATIONWIDE POPULATION-BASED STUDY}

E. Yen ${ }^{1}$, D. Singh ${ }^{1}, \mathrm{M} . \mathrm{Wu}^{1}$, R. Singh ${ }^{1} .{ }^{1}$ UCLA, Los Angeles, United States of America

Background: Premature mortality is an important way to quantify disease burden. Patients with systemic sclerosis (SSc) can die prematurely of disease, however, the premature mortality burden of SSc is unknown. The years of potential life lost (YPLL), in addition to age-standardized mortality rate (ASMR) in younger ages, can be used as measures of premature death.

Objectives: To evaluate the premature mortality burden of SSc by calculating: 1) the proportions of SSc deaths as compared to deaths from all other causes (non-SSc) by age groups over time, 2) ASMR for SSc relative to non-SSc-ASMR by age groups over time, and 3) the YPLL for SSc relative to other autoimmune diseases.

Methods: This is a population-based study using a national mortality database of all United States residents from 1968 through 2015, with SSc recorded as the underlying cause of death in 46,798 deaths. First, we calculated the proportions of deaths for SSc and non-SSc by age groups for each of 48 years and performed joinpoint regression trend analysis ${ }^{1}$ to estimate annual percent change (APC) and average APC (AAPC) in the proportion of deaths by age. Second, we calculated ASMR for SSc and non-SSc causes and ratio of SSc-ASMR to nonSSc-ASMR by age groups for each of 48 years, and performed joinpoint analysis to estimate APC and AAPC for these measures (SSc-ASMR, non-SSc-ASMR, and SSc-ASMR/non-SSc-ASMR ratio) by age. Third, to calculate YPLL, each decedent's age at death from a specific disease was subtracted from an arbitrary age limit of 75 years for years 2000 to 2015 . The years of life lost were then added together to yield the total YPLL for each of 13 preselected autoimmune diseases. Results: $23.4 \%$ of all SSc deaths as compared to $13.5 \%$ of non-SSc deaths occurred at $<45$ years age in 1968 ( $p<0.001$, Chi-square test). In this age group, the proportion of annual deaths decreased more for SSc than for non-SSc causes: from $23.4 \%$ in 1968 to $5.7 \%$ in 2015 at an AAPC of $-2.2 \%(95 \% \mathrm{Cl},-2.4 \%$ to $-2.0 \%)$ for SSc, and from $13.5 \%$ to $6.9 \%$ at an AAPC of $-1.5 \%(95 \% \mathrm{Cl},-1.9 \%$ to $-1.1 \%$ ) for non-SSc. Thus, in 2015, the proportion of SSc and non-SSc deaths at $<45$ year age was no longer significantly different. Consistently, SSc-ASMR decreased from $1.0(95 \% \mathrm{Cl}, 0.8$ to 1.2$)$ in 1968 to $0.4(95 \% \mathrm{Cl}, 0.3$ to 0.5$)$ per million persons in 2015 , a cumulative decrease of $60 \%$ at an AAPC of $-1.9 \%(95 \%$ $\mathrm{CI},-2.5 \%$ to $-1.2 \%$ ) in $<45$ years old. The ratio of SSc-ASMR to non-SSc-ASMR also decreased in this age group (cumulative $-20 \%$, AAPC $-0.3 \%$ ). In $<45$ years old, the YPLL for SSc was 65.2 thousand years as compared to 43.2 thousand years for rheumatoid arthritis, 18.1 thousand years for dermatomyositis, 146.8 thousand years for myocarditis, and 241 thousand years for type 1 diabetes. Conclusion: Mortality at younger ages ( $<45$ years) has decreased at a higher pace for SSc than from all other causes in the United States over a 48-year period. However, SSc accounted for more years of potential life lost than rheumatoid arthritis and dermatomyositis combined. These data warrant further studies on SSc disease burden, which can be used to develop and prioritize public health programs, assess performance of changes in treatment, identify high-risk populations, and set research priorities and funding.

References:

[1] Yen EY....Singh RR. Ann Int Med 2017;167:777-785.

Disclosure of Interests: None declared

DOI: 10.1136/annrheumdis-2020-eular.2270

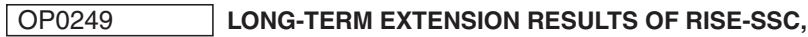 A RANDOMIZED TRIAL OF RIOCIGUAT IN PATIENTS WITH EARLY DIFFUSE CUTANEOUS SYSTEMIC SCLEROSIS (DCSSC)}

D. Khanna ${ }^{1}$, J. Pope $^{2}$, M. Matucci-Cerinic ${ }^{3}$, M. Kuwana ${ }^{4}$, C. Denton ${ }^{5}$, Y. Allanore ${ }^{6}$, M. Wosnitza ${ }^{7}$, M. E. Truchetet ${ }^{8}$, G. Szücs ${ }^{9}$, W. Stevens ${ }^{10}$, V. Steen ${ }^{11}$, C. Stagnaro ${ }^{12}$, V. Smith ${ }^{13}$, R. Silver ${ }^{14}$, E. Schiopu ${ }^{15}$, V. Riccieri ${ }^{16}$, F. Kramer ${ }^{7}$, S. Johnson ${ }^{17}$, O. Ishikawa ${ }^{18}$, T. Ishii ${ }^{19}$, E. Hachlla ${ }^{20}$, E. De Langhe ${ }^{21}$, L. Czirják ${ }^{22}$, R. Bečvárí ${ }^{23}$, T. Atsumi ${ }^{24}$, O. Distler ${ }^{25} .{ }^{1}$ Univ Michigan, Ann Arbor, United States of America; ${ }^{2}$ Univ Western Ontario, Ontario, Canada; ${ }^{3}$ Univ Florence, Florence, Italy; ${ }^{4}$ Nippon Med School, Tokyo, Japan; ${ }^{5}$ UCL, London, United Kingdom; ${ }^{6}$ Paris Descartes Univ, Paris, France; ${ }^{7}$ Bayer AG, Wuppertal, Germany; ${ }^{8}$ Bordeaux Univ Hosp, Bordeaux, France; ${ }^{9}$ Univ Debrecen, Debrecen, Hungary; ${ }^{10}$ St Vincent's Hosp, Melbourne, Australia; ${ }^{11}$ Georgetown Univ, Washington, United States of America; ${ }^{12}$ Univ Pisa, Pisa, Italy; ${ }^{13}$ Ghent Univ Hosp, Ghent, Belgium; ${ }^{14}$ Medical Univ South Carolina, Charleston, United States of America; ${ }^{15}$ Michigan Medicine Univ Hosp, Ann Arbor, United States of America; ${ }^{16}$ Sapienza Univ Rome, Rome, Italy; ${ }^{17}$ Univ Toronto, Ontario, Canada; ${ }^{18}$ Gunma Univ, Maebashi, Japan; ${ }^{19}$ Tohoko Univ, Sendai, Japan; ${ }^{20}$ Hôpital Claude Huriez, CHU, Lille Univ, Lille, France; ${ }^{21}$ Univ Hosp Leuven, Leuven, Belgium; ${ }^{22}$ Univ Pécs Medical School, Pécs, Hungary; ${ }^{23}$ Charles Univ, Prague, Czech Republic; ${ }^{24}$ Hokkaido Univ, Sapporo, Japan; ${ }^{25}$ Univ Hosp Zurich, Zurich, Switzerland

Background: RISE-SSc (NCT02283762) was a multicenter Phase Ilb trial of riociguat in pts with early (duration $\leq 18$ months) dcSSc and modified Rodnan skin score (mRSS) 10-22 units. Pts were randomized double-blind to placebo or riociguat $0.5-2.5 \mathrm{mg}$ t.i.d. for 52 weeks. The primary endpoint, mRSS change from baseline to Week (Wk) 52, did not reach statistical significance $(p=0.08$, riociguat vs placebo), but there were favorable trends in some other outcomes. Objectives: To present open-label long-term extension (LTE) results of RISE-SSc.

Methods: Pts who completed Wk 52 of double-blind therapy could enter LTE on riociguat. Endpoints included mRSS, adverse events (AEs), and serious AEs (SAEs)

Results: Of 60 pts randomized to riociguat and 61 to placebo, 42 (riociguat-riociguat group) and 45 (former placebo group), respectively, entered LTE. At LTE start, mean \pm SD mRSS was $16.4 \pm 3.2$ and $16.3 \pm 4.2$ units, and mean disease duration was $8.9 \pm 7.8$ and $8.9 \pm 5.8$ months, in the riociguat-riociguat and former placebo groups, respectively. Other demographics/disease characteristics were also comparable. Median duration of riociguat treatment was $1092 \mathrm{~d}$ in riociguat-riociguat pts and $649 \mathrm{~d}$ in former placebo pts. Throughout the study, mRSS decreased in both groups (Figure 1). From Wk 52 to last visit, mRSS fell by $-3.02 \pm 5.51$ in riociguat-riociguat patients and $-3.96 \pm 5.43$ in former placebo pts. Rates of $m R S S$ regression (decrease by $>5$ units and $\geq 25 \%$ from Wk 52 to last visit) and of \% declines in mRSS were similar in the two groups (Figure 2). mRSS progression (increase by $>5$ units and $\geq 25 \%$ from Wk 52 to last visit) occurred in 1 pt $(2 \%)$ in each group. During the entire study, rescue therapy agents were used in $15(36 \%)$ riociguat-riociguat pts and $17(38 \%)$ former placebo pts. AEs were reported from Wk 52 to last visit in 82 pts (94\%): 40 (95\%) riociguat-riociguat and 42 (93\%) former placebo. Most common AEs overall: nasopharyngitis (24\%), gastroesophageal reflux disease (17\%), diarrhea (15\%), and hypotension (14\%). AEs of special interest (dizziness, postural dizziness, or hypotension) occurred in 5 riociguat-riociguat pts (12\%) and 4 former placebo pts $(9 \%)$. SAEs were reported in $21(24 \%)$ pts: 10 (24\%) riociguat-riociguat pts and $11(24 \%)$ former placebo pts, with no SAE reported in $>1$ patient, no SAEs of special interest, and no deaths.

Conclusion: During LTE riociguat treatment, mRSS decreased in both groups from Wk 52 onwards and mRSS progression was uncommon. Riociguat had acceptable safety, similar to the main study, with no new safety signal. 